\title{
SUPPRESSING BORING BAR VIBRATIONS BY PARAMETRIC EXCITATION
}

\author{
Ahmida Rezig* \& Mohammed Ouali** \\ *Department of Mechanical, Ammar Thelidji University, Laghouat, Algeria, BP 5304, El- \\ Makam Laghouat, 03000, Algeria. Tel.: (213) 0662605810 \\ **Department of Mechanical, Saad Dahlab University, Blida, Algeria, Blida, Algeria, Tel.: \\ (213) 0661597102. \\ E-mail: rezigah_go@yahoo.fr; oualimohammed@yahoo.fr
}

\begin{abstract}
:
Machine tool chatter causes an instability that can occur in tool-workpiece-machine system. Hence surface roughness and tool wear in metal cutting processes. This instability caused by interaction between current tool positions and surface left by previous tool passes. Many methods have been developed to minimize the effects of regenerative chatter in machine tools and to enhance the damping capability minimizing the loss in static stiffness through implementation of device uses PZT actuators or MR fluid. The possibility of suppressing selfexcited vibrations of boring process using parametric excitation is discussed. We consider a two-mass system of which the main mass is excited by a self excited force (cutting force). A single mass which acts as a dynamic absorber is attached to the main mass and, by varying the stiffness between the main mass and the absorber mass, represents a parametric excitation. Cutting experiments with an excitation current of different waveforms and diverse frequencies show that chatter can be significantly suppressed by the effect of parametric excitation.
\end{abstract}

Key Words: Parametric Excitation, Chatter, Boring Process, Stability

\section{Nomenclature}

$\begin{array}{ll}\omega_{F} & \text { excitation frequency }(\mathrm{Hz}) \\ T & \text { period [s] } \\ \Omega & \text { natural frequencies of boring bar [rad/s] } \\ M_{R}, M_{B} & \text { Mass of rod and boring bar respectively } \\ K_{B} & \text { boring bar stiffness }(\mathrm{N} / \mathrm{m}) \\ K_{N} & \text { rubber stiffness }(\mathrm{N} / \mathrm{m}) \\ K_{P E} & \text { single stiffness element }(\mathrm{N} / \mathrm{m}) \\ F_{C} & \text { cutting force magnitude }(\mathrm{N}) \\ C_{B}, C_{R} & \text { damping of boring bar and rod respectively } \\ b & \text { chip width }(\mathrm{m}) \\ K_{s} & \text { cutting force coefficient }\left(\mathrm{N} / \mathrm{m}^{2}\right) \\ h & \text { instantaneous chip thickness }(\mathrm{m}) \\ h_{0} & \text { normal chip thickness }(\mathrm{m}) \\ M, C, K & \text { mass matrix, damping matrix, stiffness matrix } \\ P_{S} & \text { static load }(\mathrm{N}) \\ P_{t} & \text { time component of the load }(\mathrm{N})\end{array}$

\section{INTRODUCTION}

Removing high volumes of material in shorter time as well as obtaining the right quality from the first part produced are goals that one would like to achieve. Tooling systems, and especially cantilever tools, and cantilever structural units of machine tools are the least rigid components of machining systems and therefore the most prone to vibration that could lead to cutting instability. The objective of this paper is to implement efficient damping devices 
based on identification of parametric models describing the dynamic stability of machining systems. The present paper focuses on the design and the dynamic analysis of damped boring bar used in internal turning. In order to understand the principle for design of efficient damping systems it is necessary to understand the dynamic behavior of machining systems. Machining systems may be represented by a closed loop system comprising the machine tool elastic structure, the machine tool structure including tool, tool holder, workpiece etc., and the cutting process, turning, milling etc., the interaction between the machine tool's elastic structure and the cutting process describes the behavior of the machining system. This behavior directly affects the process accuracy.

\subsection{State of the art in parametric excitation}

All mechanical engineering systems which undergo oscillatory motion are often described by a finite set of governing differential equations. If for some reason one or more parameters, such as the mass, damping, stiffness, appear as time varying coefficients in these equations, the system may be said to be subjected to parametric excitation, and this phenomenon is then called parametric vibration[1-3].

This is one aspect that parametric vibration differs from the familiar forced vibration, whose coefficients in the equations are all constant. The other difference between these two vibrations is that parametric vibration might occur in directions normal to the excitation, while forced vibration is understood to appear only in directions parallel to the excitation.

Later investigations incorporated such factors as different elastic members, such as beams, rods, bars, etc; and various boundary conditions, inertia parameters, and different forms of excitations. Dugundji and Mukhopadhyay (1973) carried out a study on a horizontally-orientated cantilever beam subjected to vertical harmonic excitation of its base [4].

The equations of motion were reduced to Mathieu equations. This caused combination resonance, with primary instability regions defined when exciting such that $\omega_{\mathrm{F}}=\Omega_{1}+\Omega_{2}$ and $\omega_{\mathrm{F}}=\Omega_{2}+\Omega_{3}\left(\omega_{\mathrm{F}}\right.$ is the excitation frequency. $\Omega_{1}, \Omega_{2}$ and $\Omega_{3}$ are natural frequencies of the first bending, the second bending and the torsional modes of the beam, respectively), with the two modes oscillated simultaneously, each at its own frequency $\Omega_{1}, \Omega_{2}$ and $\Omega_{3}$. This showed that lateral bending and twist could be excited by vertical base motion. Cartmell and Robert (1987) theoretically and experimentally investigated the response of a vertically-orientated cantilever beam with an attached end mass system subjected to a parametric excitation [5]. They also found that the parametric excitation promoted a sum-type combination resonance involving two modes of vibration. Analytical studies of the parametrically-excited pendulum can be traced back to the work of Leven and Koch (1981). They identified that the pendulum behaved in a chaotic way under certain parameter intervals. Miles (1985) analyzed the response of a double pendulum system under a parametric excitation through the vertical translation of the pivot of the slower pendulum. In his work, the ratio of the natural frequencies of the two normal modes approximated 2. His results showed that when the lower mode was excited by a principal parametric resonance, the resulting motion may be either a simple (rigid-body) translation of the entire system or coupled oscillations of the pendulums superimposed on such a translation [7]. Watt and Cartmell (1994) designed a single-degree-of-freedom parametric oscillator, onto which was mounted a simple mechanical power take-off device, so that the axial input motion could be converted to a parametric resonance in the torsional system to transfer energy to an external load, hereby acting potentially as a mechanical power transmission. Cartmell and Roberts (1988) presented a study of a L-shape beam structure. The structure comprised a horizontal beam and a smaller vertical beam. The horizontal beam was clamped at one end and the free end was coupled to the vertical beam. Two combination resonances, involving the fundamental and second bending modes and the fundamental torsion mode of the structure, could be generated when the external excitation of the support was at a frequency in the region of the second bending mode frequency of the system when it oscillated in the least stiff plane. 
The growth of the amplitude of the vibrations during parametric excitation is provided by the force that periodically changes the parameter. Parametric resonance is possible when one of the following conditions for the frequency $\omega$ (or for the period $T$ ) of modulation is fulfilled;

$$
\omega=\frac{2 \omega_{0}}{n}, \quad T=\frac{n T_{0}}{2}, \quad(n=1,2,3, \ldots)
$$

In other words, parametric resonance occurs when the parameter changes twice during one period, once during one period, twice during three periods, and so on. However, the maximum energy transfer to the vibrating system occurs when the parameter is changed twice during one period of the natural frequency. In this paper, we are interested in the case, in which parametric force has the frequency of twice the natural frequency of the system.

\subsection{Methods of stability analysis of parametrically excited system}

The governing equations for parametrically excited systems are second order differential equations with periodic coefficients, which have no exact solutions. The researchers for a long time have been interested to explore different solution methods to this class of problem. The two main objectives of this class of researchers are to establish the existence of peri odic solutions and their stability. When the governing equation of motion for the system is of Matheiu-Hill type, a few well known solution methods those are commonly used are, method proposed by Bolotin based on Floquet's theory, perturbation and iteration techniques, the Galerkin's method, the Lyapunov second method and the asymptotic technique by Krylov, Bogoliubov and Mitroploskii.

\section{DESIGN OF BORING BAR}

\subsection{Concept}

Our study is based on a model for the suppression of chatter vibrations of boring bar by a dynamic absorber with parametric excitation formulated in [11]. Consider a two mass system consisting of a main mass $M_{B}$ which is subject to self-excitation vibration and an absorber mass $M_{R}$ which is attached to the main mass by a spring element, see Figure 1 . The elastic mounting $K_{P E}$ of the absorber mass is a combination of a spring and a device operating such that the stiffness $K_{P E}$ is changed periodically. Damping is represented by the linear viscous damper $C_{R}$. The main mass $M_{B}$ is supported by a spring with constant stiffness $K_{B}$; it has a linear viscous damper with damping parameter $C_{B}$. In actual constructions one usually has $M_{R}<M_{B}$.

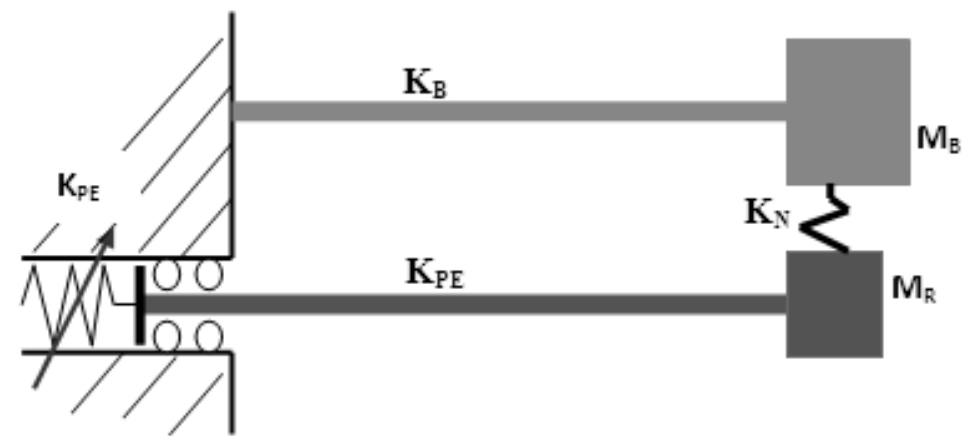

Figure 1: Interaction between Self-excited and Parametric Vibrations.

The boring process generated self-excited force is acting on the main mass $M_{B}$. The displacements of mass $M_{B}$ and mass $M_{R}$ are denoted by the coordinates $\mathrm{x}_{B}$ and $\mathrm{x}_{R}$, respectively. The variation of the stiffness of the absorber element is supposed to be a harmonic function with small amplitude. 


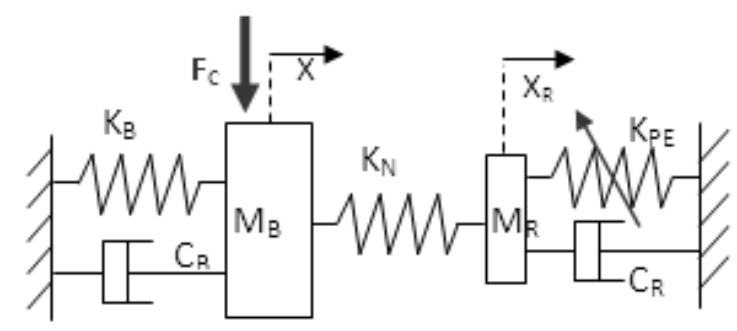

Figure 2: The schematic representation of the two mass chain system.

This system is represented by the following nonlinear equations of motion:

$$
\begin{aligned}
& M_{R} \ddot{x}_{R}+C_{R} \dot{x}_{R}+K_{R}(t) x_{R}-K_{N}\left(x_{R}-x_{B}\right)=0 \\
& M_{B} \dot{x}_{B}+C_{B} \dot{x}_{B}+K_{B} x_{B}+K_{N}\left(x_{R}-x_{B}\right)=F_{g}(\mathrm{t})
\end{aligned}
$$

where: $K_{P E}=K_{R}(t)$

$K_{P E}:$ Parametric excitation stiffnes.

with: $M$ is the mass, $C$ is the damping, $K$ is the stiffness, and the right side of the equation (2) represents the cutting force acting on the tip of the boring bar.

$$
\begin{aligned}
& M_{R} \tilde{x}_{R}+C_{R} \dot{x}_{R}+K_{R}(1+\varepsilon \cos \omega \tau) x_{R}-K_{N}\left(x_{R}-x_{B}\right)=0 \\
& M_{B} \dot{x}_{B}+C_{B} \dot{x}_{B}+K_{B} x_{B}+K_{N}\left(x_{R}-x_{B}\right)=F_{\varepsilon}(t)
\end{aligned}
$$

where: $K_{R}(t)=K_{R}(1+\varepsilon \cos \omega \tau)$

The above equations (3) and (4) can be written in matrix form as:

$$
\begin{aligned}
& {\left[\begin{array}{cc}
M_{R} & 0 \\
0 & M_{B}
\end{array}\right]\left\{\begin{array}{l}
\ddot{x}_{R} \\
\tilde{x}_{B}
\end{array}\right\}+\left[\begin{array}{cc}
C_{R} & 0 \\
0 & C_{B}
\end{array}\right]\left\{\begin{array}{l}
\dot{x}_{R} \\
\dot{x}_{B}
\end{array}\right\}+\left[\begin{array}{cc}
K_{R}(1+\varepsilon \cos \omega \tau)+K_{R} & -K_{N} \\
-K_{N} & K_{B}+K_{N}
\end{array}\right]\left\{\begin{array}{l}
x_{R} \\
x_{B}
\end{array}\right\}=\left\{\begin{array}{c}
0 \\
F_{o}(t)
\end{array}\right\}} \\
& F_{0}(t)=K_{s^{*}} b \cdot h(t)
\end{aligned}
$$

According to the low of regeneration [8] and [9] with the thickness of the chip can be expressed by:

$$
h(t)=h_{0}-[x(t)-x(t-T)]
$$

where $\tau$ the delay time.

The motion of an $n$ degree-of-freedom system can be represented by a system of secondorder differential equations:

$$
M \ddot{x}(t)+C \ddot{x}(t)+K x(t)=f_{c}(t)
$$

Where $x_{R}$ and $x_{B}$ are the displacement, $f_{c}(t)$ is the excitation force, $M$ (mass matrix), $C$ damping matrix) and $K$ (stiffness matrix).

The boring bar is subjected to a pulsating axial force $P(t)=P_{s}+P_{t} \cos \omega_{F} t$, acting along its lateral side. $\omega_{F}$ is the excitation frequency of the dynamic load component, $P_{s}$ is the static and $\mathrm{P}_{\mathrm{t}}$ is the time component of the load. 


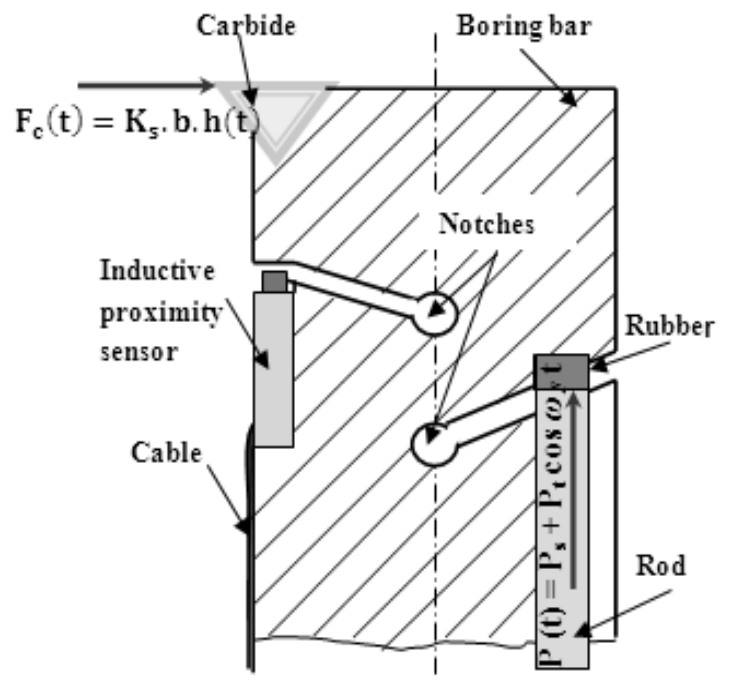

Figure 3: Boring bar with boundary conditions (fixed-free).

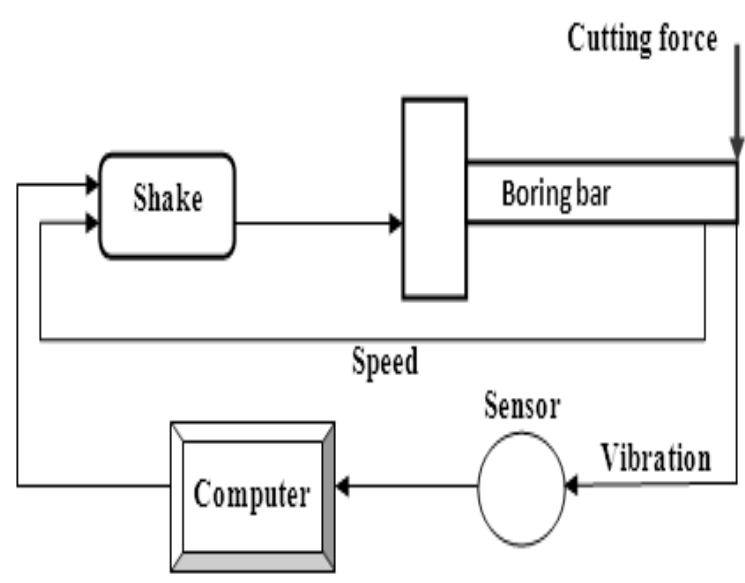

Figure 4: Block diagram of the control.

\subsection{Final design}

To maintain a high level of static stiffness, it was chosen to adapt the following solution figure 3 witch use the excitation of the active part of the tool from the back by a rod which receives parametric excitation from shaker mounted on the tool holder.

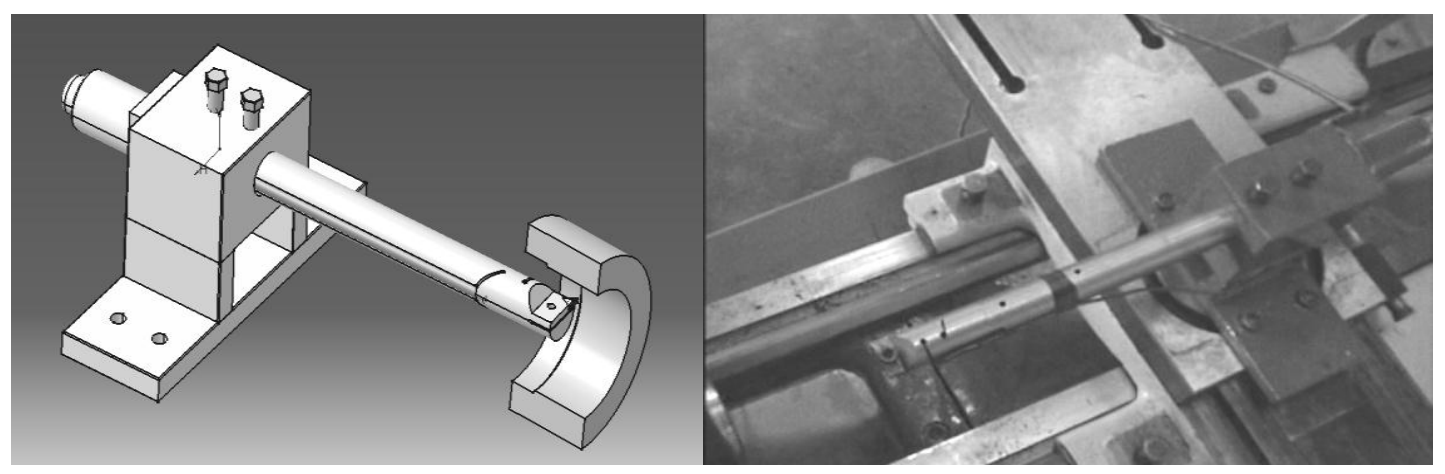

Figure 5: CAD model of the boring bar.

Figure 6: Machining configuration.

\section{PERFORMANCE EVALUATION}

\subsection{Analysis method}

The evaluation of the novel design compared to the conventional tool has been carried out in two steps: at first MOVILOG fft2 with DIVA software has been employed to produce signals and to extract the dynamic characteristics of the process machine interaction. The surface roughness has been measured after every test with a Mitutoyo Surftest 201 and correlated to the vibration signals.

\subsection{Machining tests}

The tools have been tested clamped in the same tool holder configurations as modal analysis with an overhang of $250 \mathrm{~mm}$ and a diameter of $32 \mathrm{~mm}$. Round workpieces made of XC38 with a outer diameter of $160 \mathrm{~mm}$, a inner diameter of $100 \mathrm{~mm}$ and a length of $60 \mathrm{~mm}$ were machined. The machining operations were carried out at three different depths of cut 
$a_{p}, 0.5 \mathrm{~mm}, 1 \mathrm{~mm}, 1.5 \mathrm{~mm}$. Keeping constant cutting speed $V_{c}$ at $120 \mathrm{~m} / \mathrm{min}$ and feed $\mathrm{f}$ at 0.2 $\mathrm{mm} / \mathrm{tr}$. The effect of the tool's damping ratio on the machining process is shown in figure 6 and figure 7 , where the signals produced by machining with conventional and the damped tool (under PE and without PE) respectively, are compared.

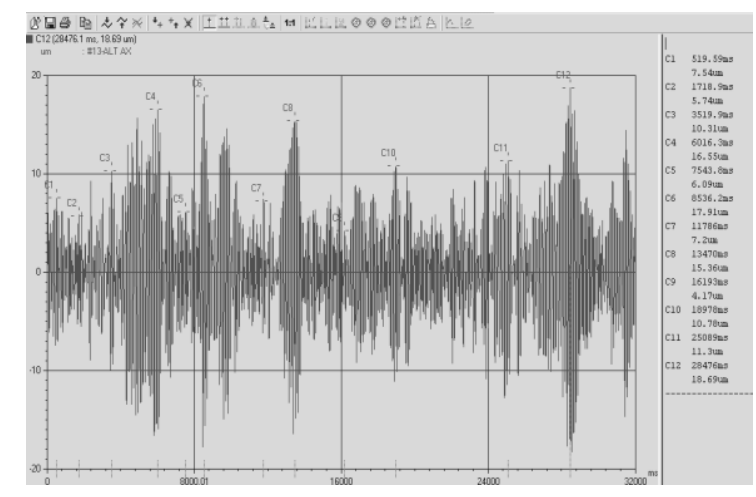

Figure 7: Signal produced by machining with conventional tool.

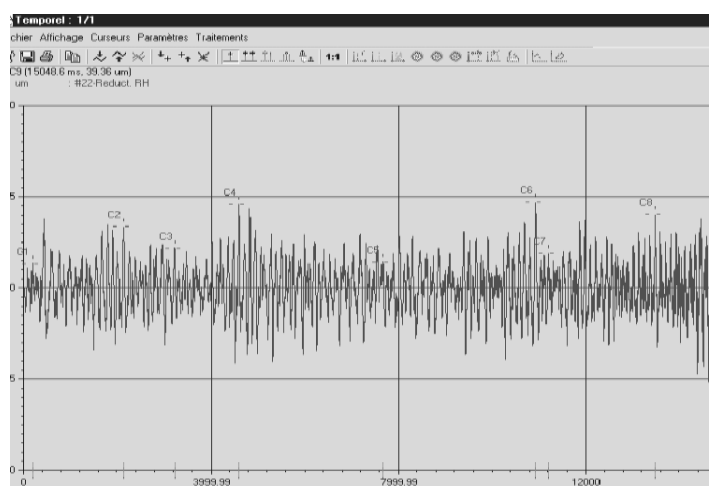

Figure 8: Signal produced by machining with damped tool.

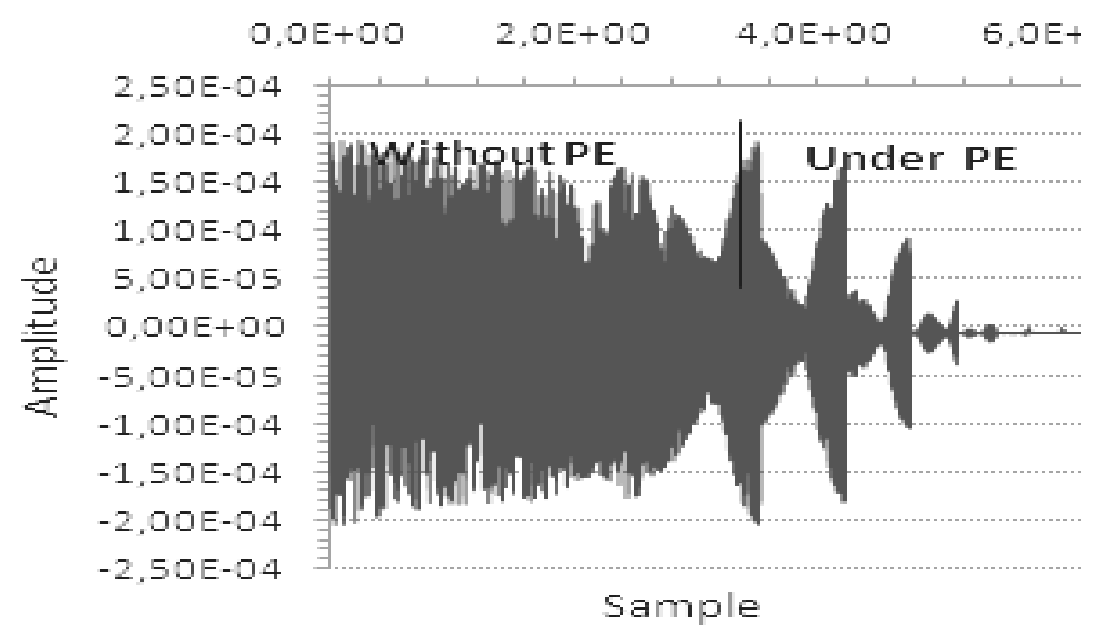

Figure 9: Time record of signal produced when machining at $\mathrm{v}_{\mathrm{c}}=120 \mathrm{~m} / \mathrm{min} \mathrm{f}=0.2 \mathrm{~mm} / \mathrm{rev}$ and $a_{p}=1 \mathrm{~mm}$.

\section{SURFACE ROUGHNESS}

The surface finish produced by the conventional tool is of much lower quality if compared to the one produced by the damped tool with parametric excitation. Figure 9 shows the surface profile taken after machining at $1 \mathrm{~mm}$; the conventional tool is not able to perform in stable conditions and therefore the surface profile is disturbed by the chatter marks. 


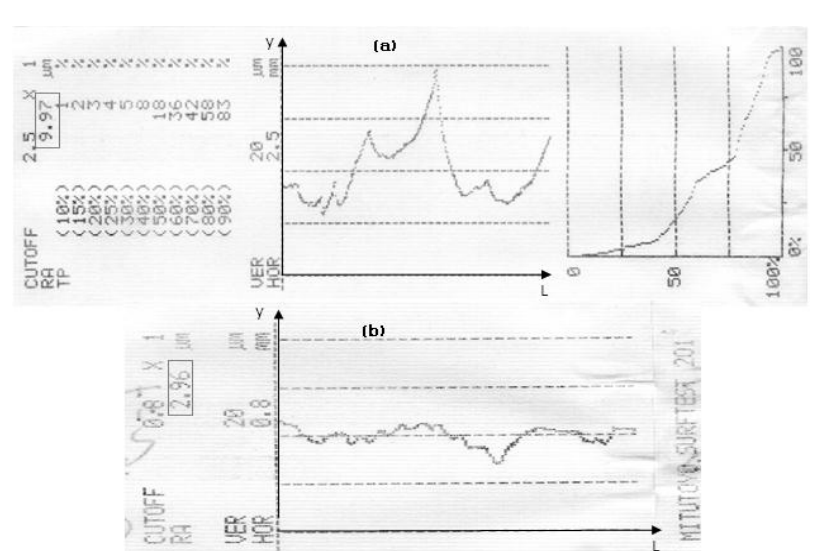

Figure 10: Surface roughness scans.

a) Conventional tool,

b) Damped tool with $P E$; after machining at $V_{c}=120 \mathrm{~m} / \mathrm{min}, f=0.2 \mathrm{~mm} / \mathrm{rev}$, and $a_{p}=1 \mathrm{~mm}$.

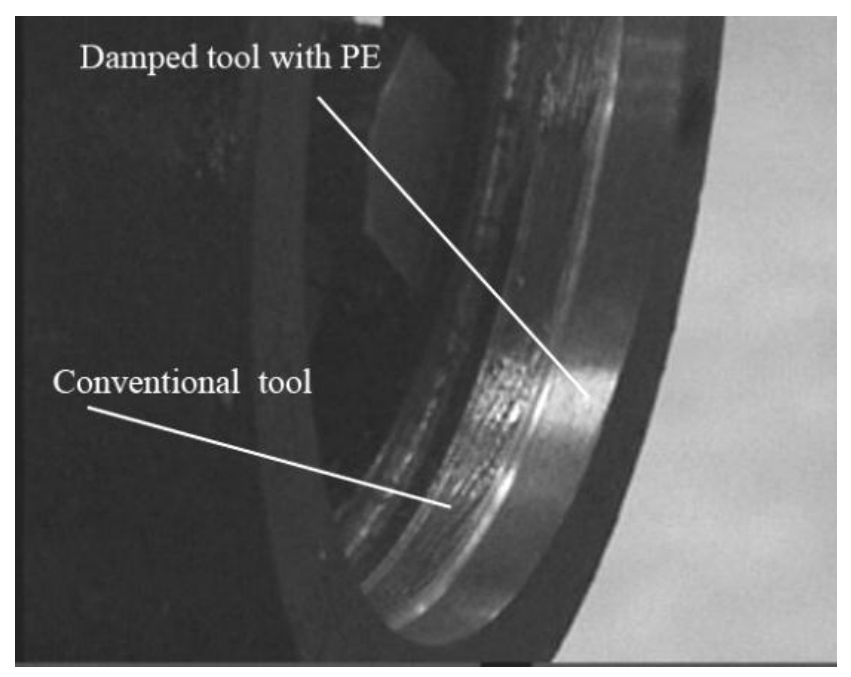

Figure 12: Photo of surface finish with chatter marks produced by conventional tool and high quality surface finish with damped tool with PE.

\section{CONCLUSION}

The stability of the linerized boring bar attached element system is investigated by a numerical simulations indicate that parametric stiffness excitation has a better effect on regenerative chatter suppression. The striking advantage of this application presented is the fact that parametric excitation only needs an open loop control system. This might be very advantageous for other applications, since it will save the cost, weight and energy for sensors and controllers, which might be very important in certain applications. The results of experiments carried out show that this new design of boring bar can be a good solution to suppress chatter vibrations.

\section{ACKNOWLEDGMENT}

I would like to express my gratitude to Laghouat University, Algeria, and maintenance management company (Sonatrach) for giving me the opportunity to carry out this research. 


\section{REFERENCES}

[1] R. A. Ibrahim, (1978). Parametric vibration Part III: Current problems (1). Shock and Vibration, Digest 10, 3, 41-57

[2] A. H. Nayfeh, and D.T. Mook, (1979). Nonlinear Oscillations. New York: Wiley

[3] M. P. Cartmell, (1990). The equations of motion for a parametrically excited cantilever beam. Journal of Sound and Vibration, 143 (3), 395-406.

[4] A. Tondl, (1978). On the Interaction between Self-excited and Parametric Vibrations, Monographs and Memoranda N0.25, National Research Institute for Machine Design, Prague

[5] M. P. Cartmell, and J. W. Roberts, (1987), Simultaneous Combination Resonances in a Parametrically Excited Cantilever Beam. Strain, 23 (3), 117-126.

[6] R. W. Leven, and Koch, B. P. (1981), Chaotic behavior of a parametrically excited damped pendulum. Physics Letters, 86A, 71-74

[7] J. Miles, (1985). Parametric excitation of an internally resonant double pendulum, Journal of Applied Mathematical Physics, 36, 337-345

[8] S. A. Tobias, (1965). Machine tool vibration, London

[9] L. Daghini, (2008). Theoretical and Experimental Study of Tooling Systems. Stockholm, Sweden: Royal Institute of Technology, KTH Production Engineering, Licentiate Thesis

[10] Y. Zhehe, M. Deqing, (2011). Chatter suppression by parametric excitation: Model and Experiments, Journal of Sound and Vibration, 330 (13), 2995-3005 